\title{
EDITORIAL
}

\section{Challenges, controversies, breakthroughs}

B reast cancer is the second most common cancer worldwide after lung cancer, the fifth most common cause of cancer death, and the leading cause of cancer death in women. The global burden of breast cancer exceeds all other cancers and the incidence rates of breast cancer are increasing (Jemal, A. et al. CA Cancer J. Clin. 60, 277-300; 2010). In light of these grim statistics, we commissioned a special focus issue on breast cancer for the December issue of Nature Reviews Clinical Oncology to coincide with the 2010 San Antonio Breast Cancer Symposium. We commissioned a series of Reviews to cover the controversies and challenges in treating triple-negative disease, screening, staging, diagnosis and treatment of patients with BRCA mutations and the significant treatment advances in treating these tumors that are of broad interest to the general oncology community.

The heterogeneity of breast cancers makes them both a fascinating and challenging solid tumor to diagnose and treat. Triple-negative breast cancers in particular are difficult to define-this tumor subgroup lacks expression of HER2, the estrogen receptor and progesterone receptor and do not respond to hormonal therapies or HER2-targeted therapies (owing to the lack of expression of these targets) - and these tumors are associated with a poor prognosis; thus, new systemic therapies are desperately needed. Luca Gianni and coauthors review the evidence for the biology of this subtype, which shares genetic and morphologic similarities with the basal-like breast cancer subtype but also represents a biologically distinct subtype that is heterogeneous. They also discuss potential treatment options, including poly(ADP ribose) polymerase (PARP) inhibitors, which have shown promising efficacy and safety profiles in phase I and II clinical trials in patients with triple-negative breast cancer.

Alan Ashworth and colleagues describe the concept of the synthetic lethality approach in breast cancer, which forms the basis of the development of PARP inhibitors. PARP is involved in the base-excision repair of singlestrand DNA breaks, and BRCA proteins help to restore double-strand breaks that arise when single-strand breaks are not repaired. Breast cancers with a $B R C A$ mutation leave the cell susceptible such that PARP inhibition combined with this genetic defect cannot repair DNA breaks resulting in cell death-an effect not observed in normal cells because the BRCA function compensates for PARP inhibition. Importantly, BRCA deficiency and sensitivity to PARP inhibition does not seem to be restricted to a particular histology but rather the BRCA genotype. So, the use of this synthetic-lethal approach may be beneficial in other

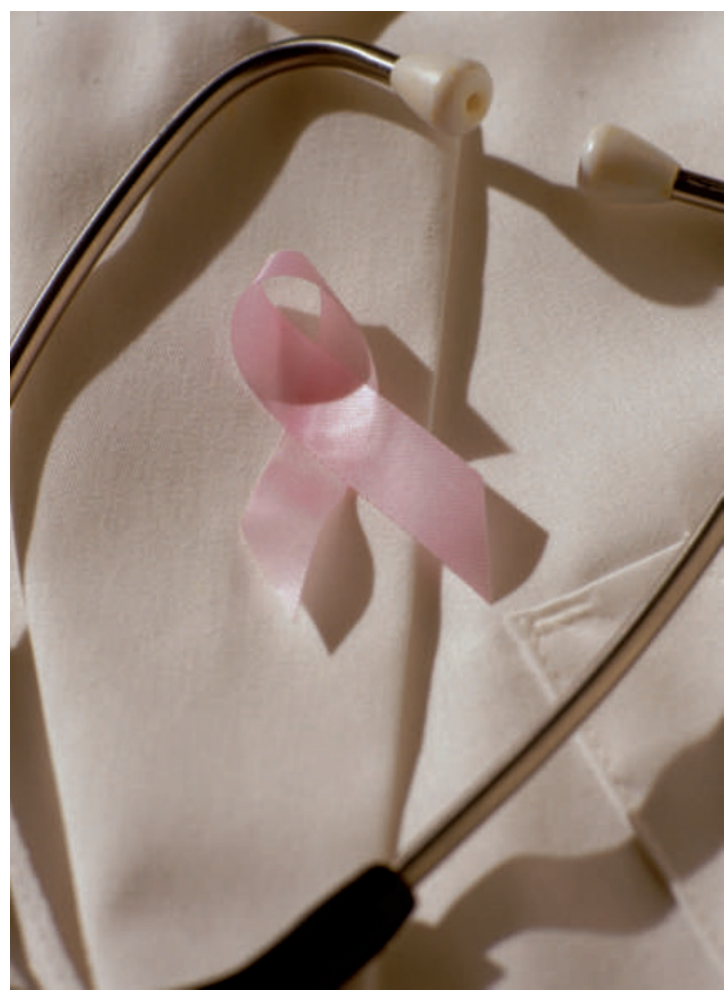

tumor types, and may provide a possible treatment breakthrough especially as many women with a $B R C A$ mutation have the triple-negative phenotype. Indeed, results of a phase II study presented by Joyce O'Shaughnessy at the recent European Society for Medical Oncology conference showed that the PARP inhibitor, iniparib, when combined with chemotherapy, significantly extended overall survival in patients with metastatic triple-negative breast cancer compared with chemotherapy alone, indicating this class of agent may provide a new treatment option for this difficult-to-treat subgroup.

Steven Narod eloquently describes screening and genetic testing in patients with $B R C A$ mutations, and explains how such testing is greatly simplified when applied to populations with founder BRCA mutations, such as Ashkenazi Jewish women with breast or ovarian cancer. The merits of primary prevention of hereditary breast cancer, including tamoxifen and oophorectomy, are also highlighted. Once a breast cancer is diagnosed, rapid genetic testing may influence the treatment strategy and allow patients and physicians to make informed decisions about the immediate surgical, radiotherapeutic or drug-treatment approach employed, as noted in a Review by Alison Trainer and coauthors. They also discuss the psychosocial aspects of
Lisa Hutchinson is the Chief Editor of Nature Reviews Clinical Oncology.

Competing interests The author declares no competing interests. 


4 ...we
may risk not
being able
to address
the most
fundamental
questions $\$ 7$

genetic testing, the additional burden associated with a diagnosis of breast cancer and the implications of how testing can be rationalized.

For the treatment of early-stage breast cancer, accelerated partial breast irradiation (APBI) has been examined as a therapeutic approach to enhance local control rates. Several randomized trials have been conducted in approximately 16,000 women to assess whether this form of radiotherapy is better than whole-breast irradiation. The recently published TARGIT trial randomized over 2,200 women and reported local recurrence rates at a median follow up of 25 months. The investigators of the trial suggest that the data indicate that APBI is safe and a single dose of radiotherapy in selected patients undergoing resection should be considered an alternative to externalbeam whole-breast irradiation. However, as early-stage breast cancer has a long natural history and recurrence rates can increase with time, Rajiv Sarin, author of a News \& Views article in this issue, cautions against the use of APBI in the absence of sufficient mature follow-up data. He explains that no significant difference in local recurrence was demonstrated at 4 years, and changes in the natural course of the disease may not be detected at this time point. Furthermore, since $20 \%$ of women in the trial received standard therapy (that is, mastectomy or wholebreast irradiation), the effect of APBI in the TARGIT trial may have been overestimated. Until further follow-up data are available, Sarin warns that premature conclusions can be counterproductive.

The articles in this issue illustrate the progress, challenges and continued controversies in the breast cancer field. One of the greatest issues in oncology is tumor heterogeneity as well as the detection and validation of biomarkers that can aid in treatment decisions. As breast cancers represent a multitude of different diseases with intratumoral and intertumoral genetic and epigenetic alterations, the next challenge will be to understand how these defects arise during disease progression and learn more about the development of mechanisms of resistance to therapies. In the future, it will be paramount to improve our scientific understanding of the carcinogenetic process so that potential biomarkers of early treatment response can be identified. Progress in understanding these disease mechanisms and potential new biomarkers is provided by Massimo Cristofanelli and coauthors, who review the role of cancer stem cells in tumor dissemination and metastasis. They discuss how genetic profiling of cancer stem cells can be used to evaluate targeted therapies and improve the clinical management of patients, which could enhance the development of personalized anticancer therapy.

As we realize the complexity resulting from the plethora of biologically distinct subtypes, conducting clinical trials to assess some of the newer agents in combination with cytotoxics and other modalities will become increasingly important. It is unlikely that there will be many circumstances where single-modality treatment will be successful. The associated challenges in undertaking new clinical trial designs and novel means of testing agents that may show efficacy in only a small patient population or in populations where the target is different to the putative target, also needs to be addressed. It might not always be possible or ethical to conduct large randomized phase III trials to test the many agents that are coming to the forefront of cancer treatment, especially if patient recruitment is based on the presence of a target or biomarker. We are still in the dark about which targets are relevant for chemotherapy response, which is important to remedy since this remains the backbone of breast cancer treatment. Should some agents in certain patient populations be approved in the absence of randomized clinical data? If we continue to conduct our trials using historical approaches, we may risk not being able to address the most fundamental questions. The oncology community needs to brace itself for a new dawn of innovative and creative means to overcome these challenges so we can witness further breakthroughs.

doi:10.1038/nrclinonc.2010.192 